\title{
Designing Requirements Engineering Research
}

\author{
Roel Wieringa \\ University of Twente \\ Department of Computer Science \\ roelw@cs.utwente.nl
}

\author{
Hans Heerkens \\ University of Twente \\ Department of Management Science \\ j.m.g.heerkens@sms.utwente.nl
}

\begin{abstract}
Engineering sciences study different topics than natural sciences, and utility is an essential factor in choosing engineering research problems. But despite these differences, research methods for the engineering sciences are no different than research methods for any other kind of science. At most there is a difference in emphasis. In the case of requirements engineering research-and more generally software engineering research-there is a confusion about the relative roles of research and about design and the methods appropriate for each of these activities. This paper analyzes these roles and provides a classification of research methods that can be used in any science-engineering or otherwise.
\end{abstract}

\section{Introduction}

In recent years, several researchers have observed that RE research tended to propose solutions without validating them $[11,48]$ Several proposals have been made for improving this situation [12, 49]. and, without having repeated our earlier investigation, an initial analysis of published RE'06 papers that we performed before writing this paper indicates that the situation has improved. However, as a community we are still grappling with some fundamental questions of engineering research design, such as what the difference between science and technology is, and which methods are appropriate for each. Confusion of technology with science leads to the treatment of technology questions like "How to add design rationale to goal-oriented RE" as if it were a scientific question to be investigated. This is methodologically unsound, for the methods to answer how-to-do questions are quite different from the methods to answer knowledge questions, and the criteria to evaluate answers to them are different too.

In this paper we define and operationalize the distinctions between technology and science and, within science, between engineering sciences and natural sciences on the other (section 3). Before we do that we discuss in section 2 the different ways in which requirements engineering is construed by members of the RE community and how this relates to the engineering sciences. In section 4 we then present a decision tree that can be used to design scientific research, and discuss whether there is any fundamental difference between using this tree in engineering science or using it in other sciences. We also use the tree to classify research approaches to validating or evaluating technology, and list the major classes of research methods that have been used in RE research.

\section{Positioning Requirements Engineering}

A convenient starting point of our analysis is the engineering cycle, which is the structure of rational action [42, 47, 49]:

- Problem investigation

- Solution design

- Design validation

- Design implementation

- Implementation evaluation

This is a logical division of tasks, and these tasks are not necessarily performed in sequence. For example, when we want to improve the use of the UML, then we must identify problems in its use and investigate what their causes are, design a solution proposal, validate that this would reduce the problems, implement this solution, and evaluate whether the implemented solution indeed has reduced the problems. These tasks can all be performed concurrently, for different versions of the improvement proposal, and ordering them in time is a matter of project management.

Three distinctions in the engineering cycle are important to identify the place of RE in technology development. First, part of the implementation task is the decomposition of the proposed solution in parts that can be composed into 
the desired solution but that can be implemented separately. We call this decomposition architecture design. For example, a proposal for improving the use of the UML could consist of providing tool support, introducing coaching, and introducing knowledge management to collect and disseminate best practices in a development organization. These solutions elements are expected to interact in such a way that the use of the UML will be improved. In general, architecture design is concerned with decomposing a solution into elements that interact in such a way that the desired properties of the solution are achieved. By contrast, RE is concerned with the tasks that logically precede architecture design in the engineering cycle, namely problem investigation, solution design and solution validation.

Note that we use the word "design" here in the sense of finding and specifying a solution to a problem. To design a solution is to specify what you will do, before you do it. So we are using "design" in the dictionary meaning of "to conceive and plan out in the mind" [41].

The second distinction to be made in terms of the engineering cycle is that there are two views of RE. Problemoriented RE consist of investigating the problem: Who are the stakeholders, what are their goals, and what are the problematic phenomena that prevent achievement of the goals. Goal-oriented RE takes this view [10, 23]. Solutionoriented RE on the other hand consists of specifying a solution to a problem: What is the desired functionality of a solution and what are its desired quality attributes. The IEEE-830 standard takes a solution-oriented view of software requirements. It is not sensible to quarrel about what the proper meaning of the word "requirements" is. But to avoid confusion in this paper we will call problem-oriented requirements goals and reserve the word requirements for desired properties of a solution.

Third, we must distinguish between a product and the process of developing and maintaining that product. IEEE830 requirements are mostly software product requirements, although the standard also includes sections to specify requirements for the development process that should deliver the product. RE research is about techniques and artifacts used in the process of RE, and about the products of this process: a requirements specification. As an illustration, the full and short papers published in the RE'06 conference treat the following topics:

- Notations to specify requirements

- Natural language

- Use case diagrams

- Feature diagrams

- Problem frame diagrams

- State machines

- Scenarios
- Tools to elicit requirements

- Video

- Mobile devices

- Kinds of requirements

- Functional requirements

- Aspects

- Quality attributes (NFRs)

- RE process techniques

- Traceability

- Evaluation techniques for solution alternatives

- Design rationale used in goal-oriented RE

- Quality criteria for requirements

Development of new techniques for the RE process is itself a two-level design process: We identify requirements and design solution techniques for a process that itself consists of identifying requirements and specifying a solution for some useful artifact. Anyone with some familiarity with compiler construction should not be confused by this.

\section{Technology, engineering science and natural science}

\section{1. $R E$ research and $R E$ technology papers}

Among the full and short papers published at RE'06 we counted 19 that proposed a solution to a problem and 6 that provide an answer to some knowledge problem. Here are some examples of papers that answer knowledge questions:

- We do not know enough about the properties of using mobile RE tools. The paper reports on some empirical studies to learn more about this.

- We know a lot about effectiveness of requirements elicitation techniques, but this knowledge is scattered over many sources. The paper aggregates empirical research results about the effectiveness of elicitation techniques.

- Not much is known about the properties of applying agile RE in standardized software processes such as commonly used in the public sector. The paper reports about lessons learned from applying agile RE in standardized processes, without claiming generality for these lessons.

And here are some examples of solution-proposal papers. 
- Late incorporation of quality attributes (NFRs) in a system leads to bad design. The paper proposes a technique to extract quality attributes as early as possible from available design documents. The claim that the techniques indeed do extract NFRs is validated by experiments and a case study reported about in the paper. The claim that early extraction using this technique improves system design is not validated in this paper.

- Requirements stated in natural language often are ambiguous, which is a problem because they can lead to products that do not match stakeholders' expectations. The paper proposes a method to detect potential ambiguity and describes an experiment that compares the performance of this method with human judgment. This experiment supports the claim that the method identifies potential ambiguity. The claim that using this method indeed this leads to improved requirements specifications is not validated in this paper.

- In practice, traceability information is not maintained because the benefits to the organization are not clear. The paper describes a project in which traceability was maintained, and argues that in this project this delivered real benefits to the company. The paper makes no claim to generality.

Some observations can be made about these examples. First, papers making knowledge claims usually have a simpler methodological structure than papers reporting about technical solutions. Papers making a knowledge claim describe some empirical procedure and then draw lessons learned, confirm or refute hypotheses, etc. The empirical procedure is usually of a known kind, with known threats to validity. The knowledge claim is evaluated by only one criterion: Is it true? Papers presenting a technical solution by contrast describe the technical solution, which is supposed to be novel and usually requires considerable explanation and comparison with related solutions to support the claim of novelty; and validation should consist of usually empirical or mathematical work that supports claims about the properties of the solution, as well as additional validation that the solution does indeed solve the problem. In addition, technical solution descriptions should also include a description of the problem to be solved to begin with, and contain a problem analysis that identifies the problemspecific criteria by which a solution should be validated.

Let us call the first kind of paper research papers and the second kind of paper technology papers. Research papers produce and justify one or more propositions that are claimed to be true. In RE research papers, this proposition is about some aspect of the RE process or about some product produced by this process. RE technology papers on the other hand, identify a problem in the RE process, propose a technique, and make some claims about it. There are two kinds of claims about a technique.

- The technique is claimed to have certain properties. For example, the authors may claim that a certain algorithm claimed to to identify quality attributes in documents, does indeed identify quality attributes, and has a certain precision. We call this kind of claim a property claim.

- The technique is claimed to solve a problem, at least to some extent. For example, the authors claim that using an algorithm to identify quality properties can be used to identify relevant quality attributes early in the requirements process and that this will improve the quality of the requirements specification and the quality of the system produced. We call this a problemsolving claim.

Problem-solving claims state that a technique $S$ in an environment $E$ will contribute to achieving goal $G$, i.e. $S \wedge E \models$ $G$ (cf. [20]). Property claims state that a technique has certain properties without referring to an environment in which the technique will be used. Both claims are part of the validation of a solution proposal. Technology papers at a conference usually do not have the space to contain all elements of a new technology description-problem identification and analysis, identification of problem-specific criteria by which to validate a solution, solution description, comparison with other solutions, validation of solution properties and validation of problem-solving power with respect to the problem-specific criteria - and very often consist of a solution description, comparison with related work, and a solution illustration.

\subsection{Science-technology interactions}

We can achieve a better understanding of science papers versus technology papers if we look at the interaction between science and technology in general. The following brief historical survey will motivate the distinction between engineering science, which is the scientific study of technology, and natural science, which is the scientific study of nature. The survey will show what the interactions between science and technology have been in other domains, and there is no reason to think that the interaction between RE science and RE technology will be any different.

We define technology as the development, production and maintenance of useful artifacts and science as the critical pursuit of knowledge. Science and technology are both systems of human activities with norms of professional behavior and values that define preference orderings over these activities. In addition, technology contain human knowledge required and produced by the activities of 
producing, developing and maintaining useful artifacts, and science contains the knowledge required and produced by the activity of critically pursuing knowledge.

The distinguishing feature of science with respect to superstition, conspiracy theory, astrology and beliefs in the return of Elvis is that science is the critical pursuit of knowledge. The scientists must bend over backwards to discuss every possible way in which his or her knowledge claim could be false [15, page 341]. The scientific research methods discussed later are particular ways of achieving this critical acquisition of scientific knowledge.

The classical view of the relationship between science and technology is linear: Basic science makes discoveries that are turned into inventions by applied science and then developed into useful artifacts by technologists [6]. As has been amply shown by historians of technology, this view is false in general $[24,35,37,25,39,40,44,50]$. Until science in its modern recognizable form came into being in the 17th century, new technology was developed without any input from science. In the 17th and 18th century, any transfer that took place was from technology to science, in the form of new observation instruments such as barometers and thermometers [5]. In the 19th century, scientists started to investigate why new technology such as steam machines, that were commercially sold and operated, actually worked [31], producing new sciences such as thermodynamics. From the end of the 19th century, industrial laboratories were founded where science and technology were in close interaction, but even here the normal mode of operation is not the linear first-science-then-technology approach [8] but a more interactive approach in which science, technology and society are continuously interacting $[1,14,17,30]$.

In this interaction, the questions asked by technologists to scientists, or by scientists about technology, are typically why some technology actually works, or what is the cause of some troublesome phenomenon in a technology. Answers have provided increased understanding of a technology and typically have helped technologists to improve performance or have helped technologists to become aware of theoretical performance limits to a technology. Other questions typically studied in engineering science are how to measure some phenomenon, how to compute certain variables, or how certain we can be that certain results obtained about models are also applicable to the technology in real life [46]. All of these are examples of knowledge transfer from science to technology. Conversely, technology transfers typically include the transfer of instruments from some technology domain to the science domain, as well as the transfer of instruments developed for scientific purposes to other domains $[25,39,13]$.

The historian of technology Layton has shown that the linear model of innovation is a fiction created at the end of the 19th century that allowed basic scientists to moti- vate the sponsoring of basic science (because it would result in commercially viable innovations) and engineers to distinguish themselves from the crafts (because it associated them with science) [36]. This mutual interest partly explains the persistence of the linear model, despite its frequent falsifications by historical studies. In addition, the linear model of innovation is very persistent among policy makers, because the OECD has collected and is continuing to collect its statistics on national research spending in terms of spending on basic research, on applied research, and on development [19]. The linear model is thus built into the OECD data collection procedure. Because future statistics should be compatible with statistics that have been collected for several decades, the OECD is not likely to change its classification. And the distinction between basic and applied science is convenient for researchers and policy makers alike, who adapt the meaning of these categories to suit their current purpose [7]. However, we should not be deluded by any of this into believing that the linear model is actually true.

To avoid any suggestion that the linear model is true we will here partition science not into basic and applied, but into engineering science and natural science. This is a distinction in terms of topics studied. We define engineering science as the critical investigation of technology and natural science as the critical investigation of anything else, including physical, chemical, biological and social reality. So for the purpose of this discussion an investigation of group dynamics is natural science just as an investigation of the properties of fluid flow is. Both kinds of phenomena appear to the researcher as given by nature.

But the distinction between the two kinds of science is fuzzy. Natural phenomena may be investigated using instruments, and can be viewed as phenomena occurring in those instruments. Conversely, technical phenomena are part of processes occurring in nature. For example, an investigation of communication patterns that occur when software engineers use a particular design notation, concerns communication phenomena that could occur in other human activities too, and could be classified as natural science or as software engineering research . But the existence of intermediate cases does not invalidate the distinction.

One advantage of making the distinction by kind of topic studied is that it makes clear that there is no master-servant relationship between the two. Results produced by studying group dynamics in software engineering projects can be used in general research in group dynamics, and vice versa. It is not the case that group dynamics research is a reference discipline and software engineering group dynamics research is an application of it. Both researches can build on results produced by the other. There is no linear assembly line of knowledge from one to the other.

A second advantage of making the distinction by topic 
is that it does not imply that engineering science is utilitydriven where natural science is curiosity-driven. In fact, all scientists are curiosity-driven, whether in natural science or engineering science. It is humanly impossible to do research and not be interested in the results. And all sponsors of research are utility-driven. It is politically or economically impossible for a research sponsor to spend public or private money on research and not have some goal with this, aiming for security, health, national status or national competitiveness [43]. But turning to the technology-side of human activities, the situation is different: Technologists, as well as their sponsors, are utility-driven because they want to contribute to solving stakeholders' problems or achieving stakeholders' goals.

A third advantage of making the distinction by topic is that it makes clear that engineering science and natural science are sciences, and use the methods of science regardless of their topic. The rise of the experimental method in the 17th century took place in disciplines associated with the crafts, alchemy and natural magic, such as mechanics, heat and magnetism, and in chemical science $[4,21,22,26,31,38]$. These were disciplines aiming to control nature by manipulating it, rather than to understand nature by reasoning about it from first principles, as classical natural philosophy did. By the end of the 18th century, technologists started investigating the properties of devices in a scientific way, performing experiments on scale models $[5,34,35]$. It is arguable that engineering as we know it today started when the experimental method was used to critically acquire knowledge about technology. This is analogous to the observation that science as we know it today started with the experimental method for critically acquiring knowledge about nature [16, page 324]. After all, the experimental method is a method for critically acquiring knowledge.

\subsection{Implications for $R E$ research and $R E$ technology}

The implications of this for RE is that we should distinguish RE technology from RE research. RE technology produces solutions for practical problems of RE. RE research investigates those solutions critically, to find out how and why they work, and what their theoretical performance limits are. In addition, RE research could investigate how to measure certain phenomena in the RE process, how to compute certain variables and how certain we can be that results acquired from simulations or experiments are applicable to the technology of interest [46].

Most papers at RE conferences make technology proposals, and if the papers come from researchers, rather than from industrial experience reports, these solutions have some claim to generality. As we saw earlier, this claim di- vides into two parts, namely (1) the technique has some properties and (2) due to these properties, the proposed technique will solve the practical problem that motivated the design. Usually, if the proposals come from practitioners, they do not have a claim to generality but they do come with an illustration that the techniques worked in particular cases. It would be a task for an RE researcher to find out why these techniques described by practitioners worked in these cases and so come up with a general theory of these techniques.

\section{Designing scientific research}

\subsection{Research as rational action}

Research is an activity to acquire knowledge critically, and this activity can be performed rationally by structuring it according to the engineering cycle.

- Research problem investigation. What is it we don't know, and why do we want to know it? Who is interested in knowing this? What do we know already? What are the research questions? What conceptual framework will we use to structure the knowledge (see figure 1)?

- Research design. Can the questions be answered nonempirically (e.g. by mathematical proof or logical argument) or should we investigate phenomena (found in nature or in technology)? If we need to investigate phenomena, what is the population of interest, how do we collect data about it and how do we analyze it? Figure 1 summarizes these choices, adds some more detail and lists possible answers to each of them.

- Research design validation. If we would perform the research as designed, would our conclusions be valid? For example, would we indeed measure the intended concepts (construct validity), would our claims about it be correct (internal validity) and could we generalize the results to the population of interest (external validity)? In which ways could our conclusions possibly be wrong?

- Do the research.

- Evaluate the results. What are the answers to our research questions? Is this a significant addition to our knowledge? Are there further questions to be answered?

Figure 1 lists well-known choices to be made in research design. Space limitations do not allow us to elaborate on this, and we here explain only one choice, namely that between modeling and sampling. When deciding how to study the 
population of interest, the researcher has a choice between selecting a subset of the population of interest to study, and modeling. If the population is not accessible (e.g. because it does not exist yet) or if accessing it would be too expensive (e.g. because it would be too dangerous or because not enough resources are available to the researcher to access the population), then we can use a model to study it. An entity $M$ is a model of a subject $S$ if studying $M$ yields knowledge about $S$ [2]. For example, we can study a scale model of an airplane wing to find properties of a wing that does not exist yet, or of a prototype of a user interface to find properties of a user interface that is not used yet, or we can study the behavior of students in a requirements engineering project to acquire knowledge about the behavior of professionals that are too expensive to have them participate in an experiment.

Models can tell us something about their subject because of some similarity between them and their subject. Showing that this similarity exists, and therefore showing what the extent of external validity of the knowledge acquired about the model is, may become a research problem in itself. For example, finding a law of similarity between wind tunnel models of real propellers and propellers in real flight, kept aeronautical engineers busy for decades in the early twentieth century [46].

\subsection{Some research methods and techniques}

Figure 2 lists some well-known research methods and how they are designed. The figure shows that we can use the tree to describe well-known research designs, and also shows that these designs still allow a large variation of measuring instruments and data analysis methods. More detail about research design is amply available in the literature, in particular for experimental design [28, 51] and case study research $[29,52]$. There is also some information about action research from the information systems domain $[3,33,45]$.

The decision tree is actually a classification of research techniques out of which to compose empirical research. It does not mention software engineering nor requirements engineering, or even engineering itself, so it is a general classification applicable to all empirical science. Figure 3 compares our classification with a well-known classification of techniques to validate software technology given by Zelkowitz and Wallace [53]. The comparison shows that our classification covers the same categories as the one of Zelkowitz and Wallace, and that their classification does not add novel techniques, except the technique they call assertion which, as they duly note, is not a research technique or method at all. An assertion is a statement made by a technologist that the technology that he or she designed is better in at least some respects than alternative technology.
Usually this statement is accompanied by an example to illustrate the point. Assertion belongs to the technology-side of our human activities, and is appropriate there, but not to the science-side.

On the other hand, it is the responsibility of a designer of technology to indicate which problems would be solved for which stakeholders, or which goals would be achieved, by his or her technical solution proposal. We return to this in the discussion at the end of this paper.

\subsection{Engineering research methods}

If all research methods that can be used in engineering sciences can also be used in natural sciences, and vice versa, then what is so special about engineering sciences other than the distinction that engineering sciences study technology rather than nature-a fuzzy distinction anyway? The distinction in methods is not a distinction in kind but in emphasis. Central element in this distinction is the importance of conditions of practice in technology, which can be ignored by natural scientists but not by engineering scientists. One of the first people to point out this difference between engineering science and natural science is Benjamin Isherwood, chief engineer of the U.S. Navy during the Civil War. Layton [36, page 693], quoting Isherwood, summarizes his views as follows.

But [in contrast to natural science] engineering deals with complicated situations in which the effects "are the joint production of many natural causes and are influenced by a variety of circumstances," so that engineers must be concerned with scale effects and conditions of practice.

The historian of technology Finch says it as follows:

In sharp contrast to the scientific worker who concentrates his efforts on the study of a special segment of his field, the professional engineer must understand and give due consideration to a wide range of pertinent factors. These include not only the relative costs, qualities, and special advantages of various materials and a knowledge of available resources of labor and equipment, but a careful analysis and appraisal of present and future economic and social needs. [16, page 331].

The professional engineer in this case includes both the practicing technologist as well as the engineering researcher investigating technology.

In an analysis of the differences between research in combustion technology (which is engineering science) and thermodynamics and fluid dynamics (which are natural sciences), Küppers has this to say about conditions of practice: 


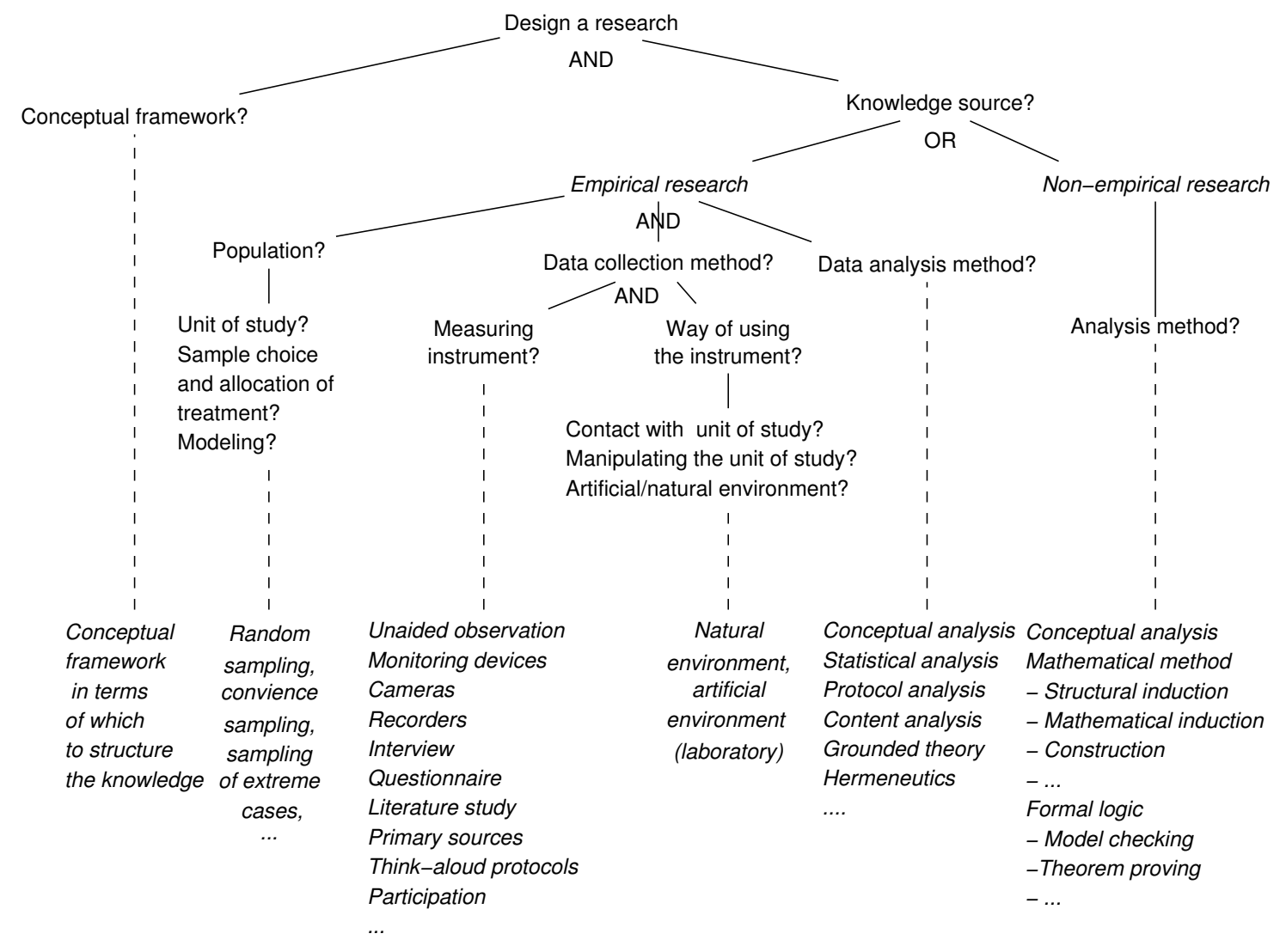

Figure 1. Choices to be made in research design. Example choices are listed in italics.

\begin{tabular}{|c|c|c|c|c|c|}
\hline & Population & Instrument & $\begin{array}{l}\text { Artificial } \\
\text { environ- } \\
\text { ment }\end{array}$ & $\begin{array}{l}\text { Manipula- } \\
\text { tion }\end{array}$ & $\begin{array}{l}\text { Data ana- } \\
\text { lysis me- } \\
\text { thod }\end{array}$ \\
\hline $\begin{array}{l}\text { Laboratory expe- } \\
\text { riment }\end{array}$ & $\begin{array}{l}\text { Relevant sample } \\
\text { (random or non- } \\
\text { random) }\end{array}$ & $\begin{array}{l}\text { Any, except instruments that collect } \\
\text { real-life data, such as primary sources } \\
\text { or participation }\end{array}$ & Yes & Yes & Any \\
\hline Simulation & Model & $\begin{array}{l}\text { Any, except instruments that collect } \\
\text { real-life data, such as primary sources } \\
\text { or participation }\end{array}$ & Yes & Yes & Any \\
\hline Field experiment & $\begin{array}{l}\text { Relevant sample } \\
\text { (random or non- } \\
\text { random) }\end{array}$ & Any, except participation & No & Yes & Any \\
\hline Field study & Relevant sample & Any, except participation & No & No & Any \\
\hline Case study & Small & Any, except participation & No & No & Any \\
\hline Ethnography & 1 & Any, including participation & No & No & Any \\
\hline Action research & 1 & Any, including participation & No & Yes & Any \\
\hline $\begin{array}{l}\text { Desk research } \\
\text { [27] }\end{array}$ & $\begin{array}{l}\text { Samples as dis- } \\
\text { cussed in the liter- } \\
\text { ature }\end{array}$ & Literature review & No & No & $\begin{array}{l}\text { Any, such } \\
\text { as content } \\
\text { analysis or } \\
\text { statistical } \\
\text { analysis }\end{array}$ \\
\hline
\end{tabular}

Figure 2. Some research design decisions made in some well-known research methods. 


\begin{tabular}{|c|c|c|}
\hline Zelkowitz and Wallace [53] & Description & Our classification \\
\hline Project monitoring & Collection and storage of project data & $\begin{array}{l}\text { Measuring instrument (primary sources) } \\
\text { (figure 1) }\end{array}$ \\
\hline Case study & $\begin{array}{l}\text { Collection of project data with a research goal in } \\
\text { mind }\end{array}$ & Research method (figure 2) \\
\hline Assertion & $\begin{array}{l}\text { The researcher has used the technique in an ex- } \\
\text { ample, with the goal of showing that the technique } \\
\text { is superior }\end{array}$ & Not a research method (not in figure 2) \\
\hline Field study & $\begin{array}{l}\text { Collection of data about several projects with a } \\
\text { research goal in min }\end{array}$ & Research method (figure 2) \\
\hline Literature search & & Measurement instrument (figure 1) \\
\hline Legacy data & $\begin{array}{l}\text { Collection of project data after the project is fin- } \\
\text { ished }\end{array}$ & $\begin{array}{l}\text { Measuring instrument (primary sources) } \\
\text { (figure 1) }\end{array}$ \\
\hline Lessons learned & $\begin{array}{l}\text { Study of lessons learned documents produced by } \\
\text { a project }\end{array}$ & $\begin{array}{l}\text { Data analysis method (conceptual analy- } \\
\text { sis)) (figure 1) }\end{array}$ \\
\hline Static analysis & $\begin{array}{l}\text { Study of a complete product (usually a program } \\
\text { and its documentation) }\end{array}$ & $\begin{array}{l}\text { Measuring instrument (primary sources) } \\
\text { (figure 1) }\end{array}$ \\
\hline Replicated experiment & $\begin{array}{l}\text { Several projects are staffed to perform a task in } \\
\text { multiple ways }\end{array}$ & Field experiment (figure 2) \\
\hline Synthetic environment experiment & $\begin{array}{l}\text { Several projects are performed in an artificial en- } \\
\text { vironment }\end{array}$ & Lab experiment (figure 2) \\
\hline Dynamic analysis & Instrumenting a software product to collect data & $\begin{array}{l}\text { Measuring instrument (monitoring de- } \\
\text { vices) (figure 1) }\end{array}$ \\
\hline Simulation & Executing a product in an artificial environment & Simulation (figure 2) \\
\hline
\end{tabular}

Figure 3. Validation methods identified by Zelkowitz and Wallace [53]. 
... in the development of furnaces it is not enough to predetermine (describe theoretically) as accurately as possible the shape of the flame, the flow pattern and the course of the reaction or the radiation pattern of the flames, one needs in addition to be sure that the flame is stable (burns in the same place), that it does not oscillate, that the furnace when turned off will not be damaged by radiation from the walls of the combustion chamber or by the flames of other surfaces and that a certain domain of regularity can be reached and maintained. These additional problem areas result from relevance criteria that hold in the economic and political domain, such as efficiency, safer operation, environmental protection. [32, page 119].

Transferring this to RE practice and research, the RE practitioner (using RE technology in practice) is not at liberty to abstract from the conditions of practice where the technology will be used. He or she has to deal with the impact of the technology on the quality of requirements, on communication with the customer and with software engineers, the speed and efficiency of the RE process, etc. And since the RE practitioner cannot ignore these conditions, ultimately the RE researchers cannot ignore them either. RE researchers can still do laboratory experiments to study a phenomenon in isolation from all the conditions of practice. This yields improved understanding of the phenomenon. But they should also study RE technology in its conditions of practice, and this motivates the use of some context-rich research methods such as case studies and action research.

Since the 19th century, engineering scientists have developed methods and techniques for approximating answers by modeling, simulation and other means. Layton shows that in the 19th century engineering scientists developed new computational techniques, including graphical techniques, for finding answers to problems for which no analytical solutions could be found [35]. He regards this as the characteristic feature of engineering science that distinguishes it from natural science:

The development of hierarchies of [computational] methods of variable rigor, along with the importance of economic factors in determining their use, served to distinguish the engineering sciences from physics where only the most rigorous methods were normally admitted. [35, page 575].

One method for approximation is to use simplified models of the subject. Where a physicist in the 19th century would analyze the behavior of a beam under stress in terms of interactions among the smallest particles that make up the beam, leading to analytically unsolvable problems, an engineering scientist would use a model that represents the beam as a large scale structure of fibers and come up with a workable answer.

Another important method of approximation when analytical solutions are not available is to use modeling and simulation. As stated before, engineering science as we know it can said to have started when Smeaton at the end of the 18th century started using scale models to study the properties of water wheels. And from the start, studying the (dis)similarity between models and the real subject has been a central concern in engineering science. Where engineering science and natural science share the critical attitude towards knowledge acquisition, engineering science emphasizes modeling, simulation and approximation of answers because it cannot abstract from conditions of practice.

In the engineering cycle, a technology can be investigated before it is implemented-validation research-or after it is implemented-evaluation research. In this terminology, most methods listed by Zelkowitz and Wallace are actually evaluation methods because they study SE technology used in projects. Replicated experiments, synthetic environment experiments and simulations (figure 3) are validation methods in our terminology, because they can be used to study a technology before it is implemented. The foregoing discussion suggests that research methods that ignore conditions of practice would be less useful for the technologists that want to use the research results in their decision-making process, than research methods that do not ignore conditions of practice. Interviews of technology managers by Zelkowitz and others [54] confirm that case study research and field research, which are richer in terms of describing actual conditions of practice, is more convincing to technology managers than laboratory experiments, which tend to abstract from those conditions.

\subsection{RE research methods}

There is no reason to believe that conditions of practice of economic cost/quality trade-offs are less important in RE research than in other engineering research. It is one thing to propose the use of video to capture requirements. It is another way to actually let practicing requirements engineers use this in a cost-effective way in actual projects. And it is the later process that should interest the RE researcher. Here are some ways in which RE technology can and has been validated.

- Modeling. The researcher, who usually also is the technologist who designed the new technology, uses the proposed technology on a real-life example but in an artificial environment. For example, a past project may be redone by the researcher-technologist using the new technology. The subject of interest, RE projects in which this new technology is used, does not exist yet; 
it is modeled by the researcher-technologist by acting as if he or she were performing such a project and then studying the result of doing so. The external validity of this is low, for if the researcher-technologist succeeds using a technology, we cannot conclude that a practicing requirements engineer would use it successfully too. However, negative conclusions have external validity, i.e. if the researcher fails in using the technology developed by him- or herself, then this is strong evidence that a practicing requirements engineer would fail too.

- Laboratory or field experiments. The technology can be used by subjects in an experiment. The subjects can be students or professionals, and the environment can be artificial (laboratory experiment) or natural (field experiment). In any of these experiments, certain response variables are measured but the purpose is not to perform an RE process as part of some real-world project. The external validity of experiments depends on the extent to which it can include the conditions of practice in the experiment.

- Action research. In action research, the researcher enters a project as a consultant and uses his or her techniques to perform tasks in the project. Here, most of the conditions of practice will be present, except one: It is not an arbitrary RE practitioner whose use of the RE technology is studied, but it is the researcher, who usually is also the designer of the technology. In fact, after the project has finished, the researchertechnologist evaluates the performance of the techniques, draws lessons learned, and possibly improves the design of the techniques.

- Pilot projects. In a pilot project, the techniques are used by others in a real-world project. Certain variables are measured by the researcher (who is not participating in the project) and after finishing the project, this is used to decide by a manager whether or not to use the technology in future projects [18]. Depending on the set-up of the pilot, all relevant conditions of practice will be present, and external validity can be high.

- Case studies. In a case study, the techniques are used by others in a real-life project, just as in a pilot study, but there usually not the intention to decide about using the technology in other projects, as there is for pilot projects [52]. However, case studies have been recommended for supporting decision about technology adoption too, in which case there is no difference with pilot projects [29].

\section{Summary and discussion}

The view of engineering as the application of science, and hence the linear view of technology as applied science, is encoded in our dictionary [41]. This paper argues for an alternative view, namely that engineering is the application of scientific research methods to the study of technology, just as natural science is the application of these methods to the study of nature. The boundary between these two kinds of science is fuzzy because phenomena in technology are part of processes in nature, and because processes in nature are observed using instruments, i.e. technology. Nevertheless, there is an important distinction in the freedom that the natural scientist has, and the engineering scientists does not have, in abstracting from the conditions of practice.

In software engineering and requirements engineering, we call the design of new technology "research" and therefore have robbed research of its name, which thereby goes unrecognized. We do indeed search for a solution to a technical problem, but this does not make it research. It would be less confusing if we call papers presenting a new technology technology papers and papers presenting research results research papers. However, typically, technology papers are called technical research papers in conferences.

Technology papers present some new technology that solves some problem for some stakeholder. They need not validate this solution, but they can illustrate it in order to explain it to their readers. However, they should indicate the relevance of the problem solved to at least some stakeholders. In particular they should identify the criteria by which the solution should be evaluated and these criteria should be motivated in terms of goals of the stakeholders in the problem. This should explain why the solution, if validated, would be useful to stakeholders.

Examples of RE technology papers from RE'06 would be descriptions of the design of RE notations, or of tools to elicit requirements, or of techniques to maintain traceability, etc. The stakeholders in these techniques include, obviously, requirements engineers, and examples of goals of a requirements engineer that could be served by these techniques are improved communication about requirements, more complete requirements elicitation, improved analysis of impact of changes of requirements, etc. However, these are rationally reconstructed goals. The relevant question for technology papers is: What are the problems that practicing requirements engineers have with these techniques? What are the conditions of RE practice that accompany actual use of these techniques, and does the technique work in those conditions? RE technology papers should refer to goals of practicing requirements engineers.

Validation that a proposed solution actually satisfies the criteria derived from an analysis of stakeholder goals is a research problem and need not be done in a technology paper. 
Research papers on the other hand answer some knowledge question about some technology and do this by applying a scientific research method to answering the question. While there are as many criteria to evaluate technology proposals as there are problems solved by these solutions, there is only one criterion by which to evaluate the answer to a research question: Is it true? This obliges the writer to discuss the validity of the answer given to the research question, and to qualify the answer with a margin of certainty. In particular, the relationship to conditions of practice should be indicated.

Given the importance of conditions of practice, the question is raised how technologists and engineering researchers in general acquire knowledge of these. In other branches of technology and engineering, technologists and engineers become familiar with conditions of practice by being a member of what Constant [9] calls a community of practice, consisting of designers, developers, testers, manufacturers, maintainers and researchers of the relevant technology. Members of a community of practice may frequently switch roles and can move from consultancy to research to various roles in the technology process. We believe this is the only way the RE researchers and technologists can familiarize them with the conditions of practice of RE.

Acknowledgment. The decision tree and table of research methods benefitted from comments made by Barbara Kitchenham.

\section{References}

[1] H. Aitken. Science, technology and economics: The invention of radio as a case study. In W. Krohn, E. Layton, and P. Weingart, editors, The Dynamics of Science and Technology. Sociology of the Sciences, II, pages 89-111. Reidel, 1978.

[2] L. Apostel. Towards a formal study of models in the nonformal sciences. In H. Freudenthal, editor, The Concept and Role of the Model in the Mathematical and the Natural and Social Sciences, pages 1-37. Reidel, 1961.

[3] R. Baskerville. Distinguishing action research from participative case studies. Journal of Systems and Information Technology, 1(1):25-45, March 1997.

[4] J. Bennett. The mechanics' philosophy and the mechanical philosophy. History of Science, 24, March 1986.

[5] Böhme, V. D. Daele, and W. Krohn. The 'scientification' of technology. In W. Krohn, E. Layton, and P. Weingart, editors, The Dynamics of Science and Technology. Sociology of the Sciences, II, pages 219-250. Reidel, 1978.

[6] M. Bunge. Technology as applied science. In F. Rapp, editor, Contributions to the Philosophy of Technology, pages 19-39. Reidel, 1974.

[7] J. Calvert. What's so special about basic research? Science, Technology and Human Values, 31(2):199-220, March 2006.
[8] W. Carlson. Innovation and the modern corporation. From heroic invention to industrial science. In J. Krige and D. Pestre, editors, Companion to Science in the Twentieth Century, pages 203-226. Routledge, 2003.

[9] E. Constant. The Origins of the Turbojet Revolution. Johns Hopkins, 1980.

[10] A. Dardenne, A. v. Lamsweerde, and S. Fickas. Goaldirected requirements acquisition. Science of Computer Programming, 20(1-2):3-50, 1993.

[11] A. Davis and A. Hickey. Requirements researchers: Do we practice what we preach? Requirements Engineering, 7(2):107-111, June 2002.

[12] A. Davis and A. Hickey. A new paradigm for planning and evaluating requirements engineering research. In 2 nd International Workshop on Comparative Evaluation in Requirements Engineeering, pages 7-16, 2004.

[13] D. DeSolla Price. Of sealing wax and string. Natural History, 84(1):49-56, 1984.

[14] A. Elzinga. The new production of reductionism in models relating to research policy. In K. Grandin, N. Wormbs, and S. Widmalm, editors, The Science-Industry Nexus: History, Policy, Implications, pages 277-304. Science History Publications, 2004.

[15] R. Feynman. Surely You're Joking Mr. Feynman! Vintage, 1992.

[16] J. Finch. Engineering and science: A historical review and appraisal. Technology and Culture, 2:318-332, 1961.

[17] M. Gibbons and C. Johnson. Science, technology and the development of the transistor. In B. Barnes and D. Edge, editors, Science in Context. Readings in the Sociology of Science, pages 177-185. Open University Press, 1982.

[18] R. Glass. Pilot studies: What, why, and how. Journal of Systems and Software, 36:85-97, 1997.

[19] B. Godin. The linear model of innovation: The historical reconstruction of an analytic framework. Science, Technology and Human Values, 31(6):639-667, November 2006.

[20] C. Gunter, E. Gunter, M. Jackson, and P. Zave. A reference model for requirements and specifications. IEEE Software, 17(3):37-43, May/June 2000.

[21] J. Henry. Animism and empiricism: Copernican physics and the origin of William Gilbert's experimental method. Journal of the History of Ideas, 62:99-119, 2001.

[22] J. Henry. The scientific revolution and the origins of modern science. Palgrave, 2002. Second edition.

[23] M. Jackson. Problem Frames: Analysing and Structuring Software Development Problems. Addison-Wesley, 2000.

[24] F. Jevons. The interaction of science and technology today, or, is science the mother of invention? Technology and Culture, 17:729-742, October 1976.

[25] A. Keller. Has science created technology? Minerva, 22(2):160-182, June 1984.

[26] A. Keller. Mathematics, mechanics and the origins of the culture of mechanical invention. Minerva, 23(3):348-361, September 1985.

[27] B. Kitchenham. Procedures for performing systematic reviews. Technical Report TR/SE-0401/0400011T.1, Keele University/National ICT Australia, 2004. 
[28] B. Kitchenham, S. Pfleeger, D. Hoaglin, K. Emam, and J. Rosenberg. Preliminary guidelines for empirical research in software engineering. IEEE Transactions on Software Engineering, 28(8):721-733, August 2002.

[29] B. Kitchenham, L. Pickard, and S. Pfleeger. Case studies for method and tool evaluation. IEEE Software, 12(4):52-62, July 1995.

[30] S. Kline. Innovation is not a linear process. Research Management, pages 36-45, July/August 1985.

[31] T. Kuhn. Mathematical versus experimental traditions in the development of physical science. In T. Kuhn, editor, The Essential Tension, pages 31-65. University of Chicago Press, 1977. Reprinted from The Journal of Interdisciplinary History, 7 (1976), pages 1-31.

[32] G. Küppers. On the relation between technology and science-goals of knowledge and dynamics of theories. The example of combustion technology, thermodynamics and fluid dynamics. In W. Krohn, E. Layton, and P. Weingart, editors, The Dynamics of Science and Technology. Sociology of the Sciences, II, pages 113-133. Reidel, 1978.

[33] F. Lau. A review on the use of action research in information systems studies. In A. Lee, J. Liebenau, and J. DeGross, editors, Information Systems and Qualitative Research, pages 31-68. Chapman \& Hall, 1997.

[34] R. Laudan. Cognitive change in technology and science. In R. Laudan, editor, The Nature of Technological Knowledge. Are Models of Scientific Change Relevant?, pages 83-104. Reidel, 1984.

[35] E. Layton. Mirror-image twins: The communities of science and technology in 19th century America. Technology and Culture, 12:562-580, October 1971.

[36] E. Layton. American ideologies of science and engineering. Technology and Culture, 17:688-701, 1976.

[37] E. Layton. Millwrights and engineers, science, social roles and the evolution of the turbine in america. In W. Krohn, E. Layton, and P. Weingart, editors, The Dynamics of Science and Technology. Sociology of the Sciences, II, pages 61-87. Reidel, 1978.

[38] P. Long. Power, patronage, and the authorship of Ars. Isis, 88:1-84, 1997.

[39] J. McKelvey. Science and technology: The driven and the driver. Technology Review, pages 38-47, January 1985.

[40] N. McKendrick. The role of science in the industrial revolution: A study of Josiah Wedgwood as a scientist and industrial chemist. In M. Teich and R. Young, editors, Changing Perspectives in the History of Science, pages 274-319. Heinemann, 1973.

[41] Merriam-Webster Inc. Webster's ninth New Collegiate Dictionary, 1988.

[42] N. Roozenburg and J. Eekels. Product design: Fundamentals and Methods. Wiley, 1995.

[43] S. Slaughter and G. Rhoades. The emergence of a competitiveness research and development policy coalition and the commercialization of academic science and technology. Science, Technology \& Human Values, 21(3):303-339, Summer 1996.

[44] C. Smith. The interaction of science and technology in the history of metallurgy. Technology and Culture, 2:357-367, 1961.
[45] G. Susman. An assessment of the scientific merits of action research. Administrative Science Quarterly, 23(4):582-603, December 1978.

[46] W. Vincenti. What Engineers Know and How They Know It. Analytical Studies from Aeronautical History. Johns Hopkins, 1990.

[47] R. Wieringa. Requirements Engineering: Frameworks for Understanding. Wiley, 1996. Also available at http://www.cs.utwente/nl/ roelw/REFU/all.pdf.

[48] R. Wieringa and J. Heerkens. The methodological soundness of requirements engineering papers: A conceptual framework and two case studies. Requirements Engineering Journal, 11(4):295-307, 2006.

[49] R. Wieringa, N. Maiden, N. Mead, and C. Rolland. Requirements engineering paper classification and evaluation criteria: A proposal and a discussion. Requirements Engineering Journal, 11:102-107, 2006.

[50] G. Wise. Science and technology. Osiris (2nd Series), 1:229-246, 1985.

[51] C. Wohlin, P. Runeson, M. Höst, M. C. Ohlsson, B. Regnell, and A. Weslén. Experimentation in Software Engineering: An Introduction. Kluwer, 2002.

[52] R. Yin. Case Study research: Design and Methods. Sage Publications, 2003. Third Edition.

[53] M. Zelkowitz and D. Wallace. Experimental models for validating technology. Computer, 31(5):23-31, 1998.

[54] M. Zelkowitz, D. Wallace, and D. Binkley. Culture conflicts in software engineering technology transfer. In 23rd NASA Goddard Space Flight Center Software Engineering Workshop, December 2-3 1998. 